\title{
Setting ad assetto variabile. La chat dei sogni in psicoterapia: il caso di Adriana
}

\author{
Carmine Parrella*
}

\section{Introduzione}

Ho di fronte a me Adriana una donna di circa trent'anni vestita con una tuta attillata, che disegna le forme di un corpo minuto. Mi sembra il corpo di una preadolescente sulla soglia dei grandi cambiamenti, quelli che ridisegnano la geografia e i confini del nuovo sé attraverso scosse telluriche che elevano colline, scavano avvallamenti e consegnano alla vita un territorio completamente nuovo da abitare e vivere. Del volto vedo solo gli occhi e la fronte alta e spaziosa, il resto, come anche accade a me, è coperto dalla mascherina dentro cui scompaiono assieme ai suoi e ai miei respiri, tutte le espressioni che dovrebbero accompagnare i nostri incontri. Così anche in questa terapia ci muoveremo come ipovedenti costretti a intuire e immaginare pezzi di noi che non possiamo vedere. La cosa che mi manca di più nel mio lavoro terapeutico, al tempo della pandemia, presso il Centro di Salute mentale sono i sorrisi. Sono costretto a una terapia senza sorrisi e per quanto il sorriso sia il risultato di una frazione impercettibile del movimento di una piccolissima parte del corpo, sento questa condizione come una profonda mutilazione dell'anima. Non so se qualcuno ha scritto qualcosa sul sorriso in terapia, ma so che tutto ha inizio con un sorriso e con un pianto. Quando dopo ore di travaglio il neonato ancora coperto di muco annuncerà la sua venuta al mondo con un gemito o un pianto, troverà a accoglierlo le braccia e il sorriso della madre. Da quel momento il gioco del pianto e del sorriso diventerà parte di quel cordone ombelicale emotivo che connetterà i due sostituendo quello fisico. Dal punto di vista psicologico incomincia il passaggio dalla dimensione simbiotica a quella fusionale, la quale a sua volta prepara il terreno per il lungo processo di separazione e individuazione dove il sorriso e il pianto

\footnotetext{
*Psicologo e Psicoterapeuta presso Centro di Salute Mentale ASL Toscana Nord Ovest zona di Lucca e socio SIPRe. E-mail: carmine.parrella@uslnordovest.toscana.it
} 
diventano i segnali attraverso il quale avviene una costante sintonizzazione tra i bisogni della madre e del bambino. Il corpo, il pianto e il sorriso diventano gli strumenti e la dimensione in cui i due sé si ridefiniscono di volta in volta come appartenenti o separati.

Nel corpo dolorante che Adriana adagia nella poltroncina del mio studio non c'è traccia di quel passaggio che porta una ragazza a essere da figlia a madre, eppure Adriana è madre di una bambina di otto anni ma di quel rapporto Adriana parlerà molto poco per tutto l'arco della terapia che è tutt'ora in corso. Mi chiedo se Adriana sia riuscita a sorridere alla sua piccola quando è venuta al mondo e se quel sorriso non fosse intriso di una qualche inesprimibile tristezza, perché quando Adriana è rimasta incinta senza volerlo e senza programmarlo, qualcosa nella vita e dentro Adriana si è fermato. Mi chiedo se Adriana stessa, quando è venuta al mondo, abbia trovato il sorriso di sua madre. Adriana ribadisce spesso nel corso degli incontri che la madre non voleva avere figli. Ha accettato solo per le pressioni del padre lasciando intuire che, senza quella concessione forse il matrimonio non sarebbe durato. Le descrizioni e il modo in cui Adriana ha interiorizzato le relazioni genitoriali precoci, mi ricordano la scimmietta del famoso esperimento di Harlow. La madre viene descritta da Adriana come la scimmietta di filo di ferro che ha il biberon e a cui Adriana si attacca per prendere il latte e il padre la scimmietta finta di peluche e senza biberon con cui passa il resto del tempo per trovare calore e sicurezza. A volte Adriana sembra rannicchiarsi nella poltroncina come se volesse tornare ad essere un feto. Anche nella poltroncina però prova dolori muscolari così intensi e forti che deve spostarsi e fare tutta la seduta da sdraiata su un materassino.

Adriana arriva preceduta dalla telefonata sconfortata del suo medico di famiglia a cui fa costanti e reiterate richieste di esami e di certificazioni. Adriana ha intensi dolori muscolari in tutte le parti del corpo, dolori diagnosticati come una forma di fibromialgia. Il dolore varia in termini di localizzazione e di intensità e le impedisce di lavorare ormai da più di un anno in un ufficio. Ha ormai utilizzato quasi tutte le forme di tutela prevista per i lavoratori e si avvicina il momento in cui ci sarà l'inevitabile licenziamento. La mancanza di chiari esiti diagnostici relativi a patologie di organo fa di lei una malata immaginaria immersa in un mondo dove nessuno crede alla sua malattia e quindi nessuno comprende e capisce il modo in cui soffre. Eppure, questo corpo un tempo era appartenuto ad un'altra Adriana. Lo si può vedere nel suo sito internet perché Adriana è stata una promessa nel mondo della musica. Le foto la ritraggono in eventi pubblici, performance interviste e premiazioni anche in prestigiosi contesti internazionali. Il corpo è nello splendore dei vent'anni, elegante e sensuale un corpo in grado di catturare l'attenzione e il desiderio, un corpo che esprime bellezza sicurezza e femminilità.

Ora quel corpo non lo vuole nessuno, o forse lo vogliono solo uomini in cerca di avventure sessuali che Adriana contatta in chat nel tentativo di rom- 
pere l'isolamento affettivo in cui è precipitata. Adriana aveva iniziato un progetto di vita con il padre della bimba, anche lui un giovane musicista con il quale aveva condiviso importanti progetti. La convivenza non era durata, Adriana si era trovata a fronteggiare una personalità narcisista con una vita sessuale sregolata e trasgressiva che non si curava di nascondere più di tanto, esponendola a costanti litigi e umiliazioni. Così era ritornata a casa a vivere con i suoi genitori mentre l'ex compagno che, continuava in modo brillante la propria carriera, metteva in atto una serie infinita di comportamenti provocatori e punitivi sottraendosi sistematicamente agli obblighi di mantenimento e costringendo Adriana ad iniziare una serie di logoranti battaglie legali.

I genitori avevano procurato ad Adriana un lavoro in un ufficio, dove si occupava della segreteria e di seguire pratiche a lei anonime. Una volta uscita dall'ufficio Adriana divideva il tempo tra le cure alla figlia e il tentativo di rimanere ancorata al mondo dello spettacolo lavorando fino a tardi allo sviluppo di nuovi progetti artistici che avrebbero dovuto riconsegnarla alla vita che aveva dovuto abbandonare, un ritmo che la stessa Adriana definirà poi come impossibile da sostenere.

I primi dolori muscolari e articolari si presentano sul lavoro impedendole di stare davanti al computer e di digitare sulla tastiera per poi sconfinare inesorabilmente in tutto il corpo. Ben presto i dolori diventano così intensi e stabili da tenerla permanentemente lontana dal lavoro. Adriana spera di ottenere il riconoscimento dell'invalidità e con una parte dei propri soldi comprare un immobile in una località turistica e vivere con i proventi di questa attività. Associa la possibilità di guarigione alla realizzazione di questo sogno. Nel corso della terapia tutti i tentativi che fa in questa direzione non hanno esito aumentando il senso di sconforto e di fallimento. Adriana trova sollievo nello yoga e si documenta attivamente su tutte le terapie alternative cercando anche di passare ad una alimentazione vegetariana. Tutti questi tentativi vengono squalificati dai genitori creando una ulteriore tensione tra loro.

Gli incontri con Adriana vertono sul conflitto con i genitori e soprattutto con la madre. I genitori vengono spesso accusati di colludere con l'ex compagno perché, nel tentativo di non esasperare il conflitto con lui, cedono spesso ai suoi ricatti e alle sue provocazioni vanificando il tentativo di Adriana di ottenere il rispetto delle regole e degli impegni concordati. Adriana descrive la madre come affettivamente assente e intrusiva. La bimba diventa il nuovo terreno di scontro tra Adriana e la madre che non approva la maggior parte delle scelte che Adriana fa per la figlia. Adriana inoltre accusa la madre di sottrarle di nascosto e buttare via oggetti che le appartengono quasi a esprimere un bisogno di controllo sulla casa o un desiderio di allontanare simbolicamente la figlia dal suo spazio vitale. Adriana per contro si difende minacciando di fare altrettanto con oggetti preziosi per la madre. Insieme al collega che la segue come psichiatra decidiamo un primo allargamento del setting includendo incontri con tutta la famiglia che co-conduciamo. In questi incontri vengo- 
no messi in scena i conflitti sopradescritti e tentiamo una mediazione e ridefinizione delle reciproche rappresentazioni. Da un lato cerchiamo di far emergere il dolore emotivo sotteso al dolore fisico di Adriana tentando di comunicare che solo curando questo dolore si potrà avere ragione del suo correlato fisico, dall'altro sottolineiamo il tentativo da parte dei genitori di prendersi cura di lei nel modo in cui loro sono al momento capaci. Evidenziamo il circuito disfunzionale tra attivazione del sistema di accudimento da parte di Adriana attraverso il dolore fisico e sconferma da parte di Adriana dei genitori nella loro capacità di dare risposte ai suoi bisogni emotivi, una forbice che da subito non sembra essere facilmente negoziabile.

Dopo circa un anno avverto che la terapia è in una fase di stallo, le narrazioni e i circuiti relazionali disfunzionali si ripetono ciclicamente senza che si presentino nel setting, sia in termini di contenuti che di processi transferali, elementi nuovi e di discontinuità che permettano di elaborare i piani emotivi dolorosi sia storici che attuali collegabili al disturbo somatoforme. Al tempo stesso sento però che Adriana ha risorse in termini di pensiero simbolico che non sono ancora riuscito ad attivare. La frustrazione di ritrovarmi costantemente ad interagire su un piano concreto fatto di dolori paralizzanti del corpo e narrazioni di genitori incapaci di fare quello che lei si aspetta aumenta il mio senso di disagio e di impotenza.

A partire da ciò propongo ad Adriana di spostare l'attenzione al mondo onirico suggerendo che in quella dimensione probabilmente, notte dopo notte, Adriana sia in grado di fare una serie di esperienze relazionali ed emotive non limitate dal dolore che ora prova nel corpo ma che siano ad esso collegate.

Per facilitare tale processo le propongo di utilizzare la 'chat dei sogni' una chat su WhatsApp, che chiaramente condividiamo in modo privato dove potrà lasciare giorno per giorno registrazioni dei vocali con il resoconto dei sogni. Io mi impegno ad ascoltare regolarmente i vocali sui sogni e lasciarle quando possibile dei brevi feedback da riprendere e approfondire nelle sedute successive.

È una variazione del setting che avevo già sperimentato con un buon esito in un altro caso complesso.

Si tratta di considerare l'assetto del setting come variabile, per introdurre modalità di relazione che favoriscano il funzionamento e l'espressione di parti del paziente che risultano difficilmente attivabili all'interno della cornice usuale. Adriana raccoglie l'invito e da quel momento incomincia a inviarmi quasi tutti i giorni anche tre sogni per volta. La maggior parte di essi sono sogni articolati che fluiscono l'uno nell'altro e solo in pochi casi si tratta di frammenti e di spezzoni isolati. Ho l'impressione che spesso i sogni siano comunicati 'a caldo' a poca distanza dal momento in cui sono stati effettuati, il tono della voce è spesso intriso dell'emotività e tono affettivo del sogno e molti sono i 'vocali' in cui Adriana mette autonomamente in relazione il contenuto dei sogni con gli accadimenti della giornata. 
Il primo sogno che Adriana consegna alla chat è il seguente e a questo ne seguiranno fino a oggi un centinaio.

\section{Sogno 1}

Ciao... Allora ti racconto del sogno stanotte, brevemente perché... eh... i dettagli... a volte sono proprio inutili, non so eh... ma comunque mi son sognata due cose ricorrenti, le sogno spesso ma non mi so neanche spiegare più di tanto il motivo, perché non sono cose di cui ho paura durante la realtà, quindi è un sogno un po' incubo un po' stressante come al solito, ansioso, di certo non rilassante... perché mi son sognata l'apocalisse, ogni tanto mi sogno l'apocalisse... qui stava per andare via la luce e l'ossigeno e ci volevano le bombolette di ossigeno... e quindi si stava cercando di venir via dalla casa, di metterci in sicurezza con la mia famiglia e di metterci su un treno... si sono comunque cose che si rifanno anche ai film perché ho visto dei film anche recentemente, dei documentari su... sui problemi climatici... e poi c'era un film che anni fa vidi che si mettevano in salvo su un treno dopo l'apocalisse, noi... noi si cercava di sopravvivere senza rimanere fermi ma muovendosi su un treno, cioè rimanendo in movimento come quel film lì. Ehm... e poi... ecco dal treno son passata a un altro sogno ricorrente che non è correlato però... tipo di, di trovarsi sui treni o sui pullman come a scuola, oppure per una gita o per altri motivi e non riuscire a... a capire qual è l'ora del treno, del pullman, di perderlo, che comunque mi succedeva spesso ma son cose che non mi succedono più da più da 15 anni e quindi... di non... di non riuscire a tornare a casa o di non riuscire ad arrivare in un posto, di perdersi, di ritrovarsi fino a sera per le stazioni o per le fermate dei pullman, di scendere alla fermata sbagliata e poi mi tocca tornare a casa a piedi, al buio, da sola con la paura... e... e la casa di cui parlo in questi due sogni è quella vecchia in realtà, io mi sogno sempre quella vecchia in cui io son cresciuta che... ma una certa nostalgia ce l'ho sempre avuta perché si stava meglio là per quanto riguarda gli ambienti, in campagna senza vicini che ti guastano l'esistenza, senza cani che ti svegliano tutti i giorni e... e praticamente... e praticamente... ehm... mi sogno con nostalgia la casa vecchia...

I sogni hanno molteplici chiavi di lettura e metodologie di lavoro in ambito clinico. Il sogno è sicuramente una grande palcoscenico dove la psiche va in scena nel tentativo di compensare e riconnettere attraverso un processo 'creativo' e non controllabile tutte le parti che nell'esperienza cosciente e diurna sono scisse, frammentate e sospese nella loro componente di desiderio o di angoscia. Questo palcoscenico onirico svolge spesso la funzione di 'laboratorio' dove la persona fa esperienza di differenti parti di sé alla ricerca di un complesso equilibrio e di una improrogabile coerenza. Questa esperienza viene poi tramutata in testo che viene offerto al terapeuta il quale ha la possi- 
bilità di iniziare a giocare con il paziente con quel testo. Uso il termine gioco perché una volta che il sogno viene tramutato in testo e offerto al terapeuta è possibile sovrapporre le due aree 'immaginative e simboliche', quelle del terapeuta stesso e quello del paziente e incominciare a scambiare e integrare le rispettive risonanze così come avviene nel gioco.

I contenuti e i temi del primo sogno sono quanto mai espliciti. Adriana è stata travolta dall'Apocalisse, non c'è più aria e non c'è più luce. Questa prima scena ci introduce alla esperienza di una catastrofe totale dove non c'è più alcun posto dove vivere e dove il corpo non può ricevere nessun nutrimento. È il modo in cui Adriana può raccontarmi il dramma della perdita del suo mondo a seguito della nascita della figlia e soprattutto del tradimento del compagno. La perdita di quel mondo è apocalittica perché non esiste dentro di sé un altro universo alternativo abitabile nulla che sia ricostruibile. Insieme alla sua famiglia deve salire su un treno per mettersi in salvo. Il treno in questione è simile al treno del film 'Snowpiercer' del regista Bong Joon-Ho, un treno che percorre in modo perpetuo un mondo privo di vita e ricoperto dai ghiacci in cui in coda stanno i miserabili e in cima i ricchi. Il treno introduce il tema della 'claustrofobia' e della 'Simbiosi' attraverso un viaggio dentro un vagone angusto e privo di spazi personali il cui scopo è la mera sopravvivenza senza una direzione reale e possibile. L'associazione con il film 'Snowpiercer' è anche la rappresentazione del declassamento avvenuto da promessa dello spettacolo a 'miserabile'. In questo viaggio non si fa menzione della figlia. La famiglia che viene sognata in questo e nella quasi totalità dei sogni, non a caso è sempre quella relativa a lei e ai suoi genitori.

Mi chiedo quanto questa scena claustrofobica sia stata proiettata nella terapia inducendomi ad trovare altro spazio allargando i confini del setting per cercare di includere qualcosa di potenzialmente vitale.

In ogni caso Adriana non può scendere da quel treno, non può tentare di fare il suo viaggio da sola. Se ci prova si ritrova incapace di orientarsi tra autobus, stazioni, orari può finire al buio in preda alla paura con l'eventualità di tornare a casa a piedi da sola esposta a pericoli. Il senso di impotenza di Adriana e la mancanza di autonomia sono totali nella vita così come nel sogno. Più i suoi genitori la spingono ad attivarsi, la richiamano alle sue responsabilità e non la difendono dagli attacchi del mondo esterno più il suo corpo si blocca si irrigidisce. L'unica possibilità di sopravvivenza sia nel sogno che nella vita reale è legata alla presenza dei genitori in una forma di legame simbiotico dove Adriana esiste ma al tempo stesso finisce per scomparire nella sua soggettività.

Il sogno restituisce alla terapia una Adriana bambina, spaventata sperduta in un mondo in cui non sa viaggiare di cui non conosce il territorio e gli snodi e che vuole disperatamente tornare alla sua casa dell'infanzia dove si sente protetta. Sognerà la casa innumerevoli volte mettendo in scena la possibilità di esistere solo all'interno di una regressione simbiotica, infatti, offre il suo 
corpo sofferente per ottenere e mantenere questo legame. A riprova di questo arriva un sogno in cui può andare a vivere in un appartamento posto sopra a quello dei suoi genitori. L'appartamento però risulta con le scale e balconi malandati e soprattutto senza ringhiere e protezioni, un appartamento dove si può rischiare di cadere nel vuoto. Successivamente Adriana invece sogna di ritrovarsi all'interno della casa dei genitori, in una stanzetta piccola e buia, uno spazio molto simile a quello di un pollaio (o di un utero penso io). Nel sogno lei è felice e soddisfatta perché finalmente ha un posto tutto suo anche se sorpresa perché si chiede come può essere soddisfatta di un posto tanto piccolo e angusto.

Lo scopo di questo contributo non è però quello di indugiare su possibili interpretazioni dei sogni o di verificare in che modo il corpo che Adriana porta in terapia e il corpo 'sognato' possano esprimere e raccontare quelle fratture del sé che sono l'obbiettivo della cura. Lo scopo principale è quello di riflettere sulla funzione che il raccontare e condividere i sogni ha all'interno della relazione terapeutica con Adriana.

Parto dalla considerazione centrale che Adriana è una figlia non voluta (almeno per quanto riguarda la sua rappresentazione) i tentativi di modulare questa rappresentazione spostando l'attenzione su tutti gli sforzi che la madre fa per sostenerla sono inutili tanto che più avanti, Adriana sogna i suoi terapeuti. Sogna che il collega psichiatra è in ritardo perché sta dando una intervista alla rai, questo la mette in eccitazione e pensa che avrebbe dovuto sistemarsi, truccarsi e cambiarsi per poter sostenere anche lei l'intervista .Il sogno prosegue poi con un vissuto di rabbia e delusione verso i suoi terapeuti che non vogliono credere a quanto la madre si comporti in modo distruttivo verso di lei e che quindi il suo venire in terapia è inutile. Nonostante questo, non essere voluta il periodo dell'infanzia è ricordato positivamente perché compensati dalla presenza del papà che ha un rapporto esclusivo con lei, anche se suscita l'invidia della madre. A proposito dell'invidia della madre Adriana ricorda che dopo cena mentre giocava felicemente con il papà la madre interveniva bruscamente per metterla a letto dicendo che era tardi distruggendo quei momenti di felicità. L'ipotesi su cui mi muovo e che il rifiuto della madre e i conseguenti vissuti di vergogna siano stati compensati dalla dimensione seduttiva eccitatoria esperita nella relazione con il papà. La dimensione eccitatoria è l'unica dimensione che permette ad Adriana di funzionare, le scelte di vita professionale nell'ambito della musica e dello spettacolo (al di là di attitudini e una naturale e sana passione per le arti) possono essere lette come il tentativo di replicare l'area eccitatoria in funzione di contrasto dell'area della vergogna e della 'miserabilità' come rappresentata nel sogno del treno.

In una prospettiva relazionale il lavoro sui sogni permette al paziente di raccontare creativamente qualcosa ma dobbiamo pensare che questo qualche cosa riguarda la relazione tra il paziente e il terapeuta: 'io ti racconto i sogni ma questi racconti riguardo te e me'. 
La chat dei sogni ha quindi la funzione di ricreare un'area fusionale 'eccitatoria' con il terapeuta che ricalca l'esperienza con il padre e con le fantasie che accompagnato le sue esperienze nel mondo dello spettacolo. Uno spazio che lei potrà riempiere di tantissimi contenuti personali e creativi e che il terapeuta porterà con sé oltre il normale orario della terapia. Come ci indica il sogno dell'intervista alla radio, attraverso la terapia lei può fantasticare che potrà tornare a esibirsi e quindi avere una attenzione esclusiva. Replicare però solo l'esperienza fusionale non è sufficiente a innescare un processo terapeutico sia nella dimensione del cambiamento che della riparazione. Bisogna staccarsi dall'area del desiderio. Una parte di Adriana mi chiede di colludere con questo aspetto, mi chiede se sarò in grado di proiettarla nell'universo bello caldo accogliente che ha perso, se sarò in grado di proteggerla dall'invidia e dall'aggressività degli altri ,ma un'altra parte di Adriana, anch'essa bene evidenziata dal contenuto dei sogni, sta chiedendo di aiutarla ad affrontare il dolore e la vergogna di essere una bimba senza madre e di essere una 'miserabile' agli occhi degli altri e di sé stessa. IL ruolo del terapeuta e della Chat dei sogni è quello di costituire uno spazio psichico fusionale dove gli elementi 'eccitatori e creativi' coesistano e si integrino con i vissuti depressivi. Si tratta di un processo psichico chiaramente molto complesso perché sperimentare in modo vero e sano l'area depressiva significa 'smontare' o 'sospendere' l'esperienza fusionale con il padre. Quando questo accade infatti il dolore fisico si intensifica, invade totalmente il corpo paralizzandola e ributtandola in un'area simbiotica. Credo che la Chat dei sogni assolva alla funzione di spazio transazionale tra gioco e realtà dove l'area del gioco, è rappresentato dall'esperienza fusionale attivata con il terapeuta dal giocare insieme con i sogni e la realtà dalla possibilità di sperimentare nella relazione con il terapeuta parti non validate o non funzionanti del sé.

Nell'ultimo sogno Adriana confeziona un pacchetto regalo per sua figlia e utilizza un bel nastro ma la madre interviene duramente dicendo che non va bene ciò che sta facendo perché quel nastro si utilizza per i regali di Natale $\mathrm{e}$ non deve abituare la bimba ad avere regali di Natale quando non lo è.

Adriana coglie del sogno solo l'aspetto persecutorio della madre che violentemente la richiama alla realtà e che la toglie da quell'area 'eccitatoria' dove Adriana può esistere solo all'interno della dimensione narcisistica e del desiderio. Nella scena del sogno si possono riscontrare però anche evidenti elementi transferali dove si proietta un'area di conflitto. La fantasia di guarigione di Adriana è legata alla possibilità di realizzare i suoi desideri. Su questo Adriana è esplicita: non posso guarire se i miei genitori non mi accudiscono in modo adeguato e se non smettono di darmi addosso, non posso guarire se il mio ex compagno non smette di provocarmi, non posso guarire se non andrò a vivere in un posto caldo e con il mare e non potrò guarire se non trovo un uomo in grado di amarmi. Il tentativo del terapeuta di negoziare questi bisogni con la realtà è visto come una operazione violenta e intrusiva che riat- 
tiva i vissuti di rabbia e di disprezzo nei confronti della madre. Penso che la chat dei sogni su WhatsApp rappresenti per Adriana lo spazio paterno, quello del desiderio incondizionato mentre la seduta reale rappresenti lo spazio materno ovvero la dimensione del limite. Immagino che una parte della trasformazione terapeutica per Adriana sia legato alla possibilità di integrare queste due dimensioni che attualmente appaiono totalmente scisse.

Un'ultima considerazione riguarda la variabile tempo nella terapia erogata nei servizi pubblici con pazienti particolarmente gravi e regrediti. Lavorare sulla dimensione fusionale per permettere un movimento regressivo con potenzialità terapeutiche anziché patologico richiede una grande quantità di disponibilità di tempo e di energie. Spesso tali percorsi terapeutici fanno affidamento a una molteplicità di figure professionali e di setting differenziati che integrandosi tra loro forniscono un'area sicura dove gli aspetti scissi e frammentati possano essere stabilmente contenuti e lentamente integrati. In assenza di questo, con pazienti quindi funzionanti socialmente ma profondamente scissi, la chat dei sogni può 'estendere' il contenitore relazionale incidendo in modo sostenibile sulla quantità di risorse che sono disponibili nell'istituzione.

Conflitto di interessi: l'autore dichiara che non vi sono potenziali conflitti di interessi.

Approvazione etica e consenso a partecipare: non necessario.

Ringraziamenti: le considerazioni su questo caso clinico sono il risultato del contributo di una riflessione comune avviata con i colleghi del Gruppo di Intervisione di Pisa, condotto dal Dr. Giuseppe Riefolo e dal mio collega con cui condivido l'intervento sul caso Dr. Guido Ambrogini. A tutti loro va il mio personale ringraziamento.

Consenso del paziente: tutti i dati personali che potessero portare ad una identificazione della protagonista sono stati modificati per mantenere e tutelare la privacy della paziente.

Ricevuto per la pubblicazione: 7 luglio 2021.

Accettato per la pubblicazione: 30 luglio 2021.

${ }^{(C)}$ Copyright: the Author(s), 2021

Licensee PAGEPress, Italy

Ricerca Psicoanalitica 2021; XXXII:587

doi:10.4081/rp.2021.587

This article is distributed under the terms of the Creative Commons Attribution Noncommercial License (by-nc 4.0) which permits any noncommercial use, distribution, and reproduction in any medium, provided the original author(s) and source are credited. 
